\title{
Control vs Content: A Systematic Review of the Social Media Research Literature
}

\author{
Fadi Almazyad \\ Worcester Polytechnic Institute \\ falmazyad@wpi.edu
}

\author{
Eleanor T. Loiacono \\ Worcester Polytechnic Institute \\ eloiacon@wpi.edu
}

\begin{abstract}
The rapid advancement of web 2.0 applications paved the way for the development of social media applications and the many features of these applications enable them to attract millions of users. Social media platforms have altered how people interact with the world and one another. Researchers in the field of Information Systems have investigated social media platforms and technologies extensively. Despite the growing number of studies on social media, however, the area remains underexamined. Given the pace and consistency of innovation in this field, identifying directions for future studies of social media-related phenomena requires a careful review of the related research completed to date.
\end{abstract}

\section{Introduction}

Social media is a powerful information and communication technology (ICT). Without it, the Arab Spring would not have happened [10]. The rapid advancement of Web 2.0 has paved the way for social media applications [47] and their numerous features, which allow them to attract millions of users. According to Hootsuite.com, 3.48 billion people currently use social media. As of December 2018, Facebook, the leading social media platform, had 2.3 billion active monthly users [15]. In addition, Twitter, another popular social media platform, has 313 million users who collectively tweet 500 million times per day [40].

Researchers in the field of Information Systems (IS) have investigated social media platforms and technologies for the past 15 years. Despite the growing number of studies on social media, however, the area still has room for more studies in different domains. Studies in the top IS journals have defined social media in various ways, including as "a group of Internet-based applications that build on the ideological and technological foundations of Web 2.0, which allows the creation and exchange of usergenerated content" [18].

Social media platforms have altered how people interact with the world and one another. These platforms and technologies provide users with innovative content-generation and -sharing experiences, enabling them to maintain existing relationships and build new ones [17]. Social mediarelated changes also extend to the enterprise levels. At the enterprise level, social media enables employees to: 1) send messages to other specific employees or broadcast messages to all workers in a given organization; 2) specify coworkers as communication partners; 3) post or edit files linked to them or their partners; and 4) view communicated connections and/or files and share information with coworkers or everyone in their organizations [24]. In addition, social media technologies allow companies to interact with their customers.

Social media companies continuously develop and implement innovative technologies that change the features and capabilities of their platforms. Given the pace and consistency of innovation in this field, identifying directions for future studies of social media-related phenomena requires a careful review of the related research completed to date.

\section{Literature Review}

The growing popularity and increasingly widespread use of social media among people and organizations have given rise to an array of interesting phenomena. These occurrences give IS researchers numerous opportunities to observe, study, and validate theories on the use of social media platforms. For example, affordance and collective action theories have been applied to examine how social media users organize to help provide lunch to poor children in China [48]. Another study applied affordance theory with connective action theory to investigate how Twitter users utilized the platform to help organize cleanup efforts during the oil spill in the Gulf of 
Mexico and pressuring oil companies to do better when drilling [44].

At the enterprise level, studies have investigated how companies use social media applications as tools to provide customer service [12] and to interact with customers to acquire feedback on future product designs [8]. How companies use social media internally to socialize employees and increase the visibility of knowledgeable employees has also been investigated [24].

A previous systematic literature review focused on classifying social media research papers into different categories following the recommendations of Mingers [32], Chen and Hirschheim [4], and Liu and Myers [26] [1]. Its classification approach was based on responses to the following two questions: 1) What types of social media papers (in terms of background paradigms, research methods, data driven vs. theory driven, and choice of social media applications) do leading IS research outlets publish? 2) What are the theoretical bases for social media research?

Another systematic literature review of social media-related literature answered different questions to classify social media research papers [3]. These questions were: 1) What subject topics are studied the most? 2) What research methods are most commonly used? 3) What are the contexts of the studies? 4) What theories are used in social network research, and what dependent variables do researchers seek to explain? Both reviews considered the theories the social media research papers used. However, they differed in other constructs and measurements.

This literature review builds on these previous literature reviews. In contrast to past reviews, however, a 2-dimension classification system was used to classify papers on social media. As Figure 1 shows, those dimensions were social media control and context.

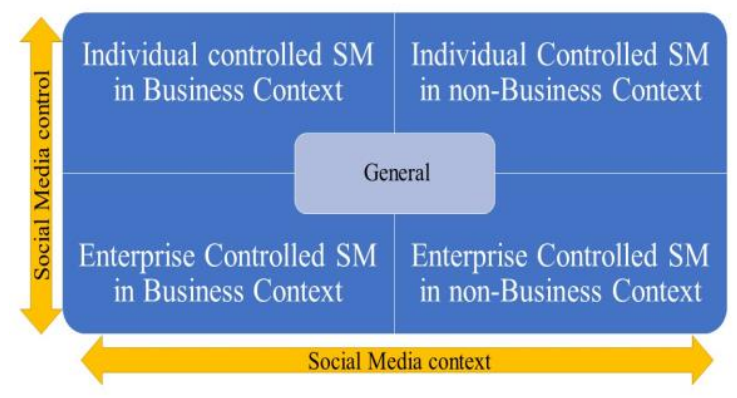

Figure. 1 Social Media Literature Review Framework

The Y-axis shows the first dimension, social media platform control, which refers to whether enterprises or non-enterprises (individuals) controlled the social media platforms in the papers under consideration. The second dimension, on the $\mathrm{X}$-axis, is context, which refers to the contexts the reviewed papers investigated (business or non-business contexts). The neutral area, labeled "General", at the center of both dimensions refers to papers that examined social media more generally-not focusing on specific contexts or controlling groups.

This classification approach facilitated the identification of both extensively researched and under-researched social media contexts and controlling groups. It will thus help researchers spot potential research gaps regarding contexts, controlling groups, or both contexts and controlling groups.

Using this approach, this study divided IS research papers on social media into the following five categories: 1) papers examining enterprise-controlled social media in business contexts; 2) papers investigating individual-controlled social media in business contexts; 3) papers analyzing enterprisecontrolled social media in non-business contexts; 4) papers considering individual-controlled social media in non-business contexts; and 5) papers examining different theories that could help explain social media phenomenon at individual and organizational levels. Sections 4-8 explain these categories in greater detail.

\section{Methodology}

This literature review uses the established guidelines for conducting an IS literature review [30]. It focuses only on highly rated IS journals based on the recommendations of Dean, Lowry and Humpherys [7] and the recommendation of Lowry, Gaskin, Humpherys, Moody, Galletta, Barlow and Wilson [27]. These recommendations agree with the recommendation of The College of Senior Scholars of the AIS regarding the Basket of Eight [43]. A total of 1896 articles from the top ranked IS journals in the basket of eight journals were searched, using the keywords "social media." After a review of the titles and abstracts of each article, a subset of 60 articles were identified as potentially relevant to the social media domain and reviewed more deeply. Table 1 shows the journals that were searched and the number of social media-related articles that were identified. Figure 2 shows the distribution of research papers by journals in each category. 


\begin{tabular}{|c|c|c|}
\hline \multirow{2}{*}{ Journal's Name } & \multicolumn{2}{|c|}{ Social Media } \\
\cline { 2 - 3 } & Total Results & Relevnce \\
\hline EJIS & 417 & 6 \\
\hline ISJ & 325 & 4 \\
\hline ISR & 381 & 14 \\
\hline JAIS & 174 & 8 \\
\hline JIT & 384 & 4 \\
\hline JMIS & 13 & 7 \\
\hline JSIS & 150 & 5 \\
\hline MIS Quarterly & 52 & 12 \\
\hline & 1896 & 60 \\
\hline
\end{tabular}

Table. 1 Result of social media search in the Basket of 8

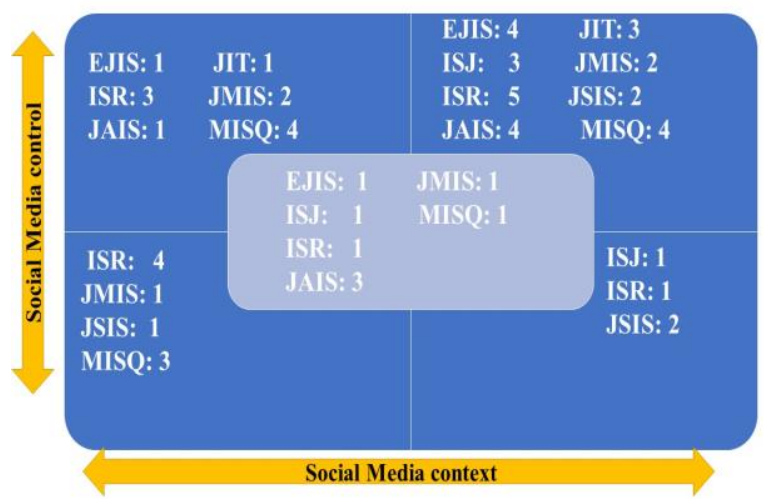

Figure. 2 Number of articles by each journal in each area

\section{Enterprise-Controlled Social Media in Business Context}

As mentioned earlier, the rapidly increasing popularity of social media has caused companies and organizations to integrate social media technologies into their business operations. Companies and organizations have begun using social media on a daily basis both internally and externally. Many research papers in top IS journals have investigated how companies have incorporated social media into their business.

In this review, papers in the "enterprise-controlled social media in business contexts" category investigated enterprises' use of social media to perform business-related activities. For example, how United States airlines use Twitter as a customer service tool was investigated [13]. This study examined this phenomenon using complaint management and service-level differentiation. It concluded that social media influence and the bystander effect may impact how airlines treat customers on social media [12].
More specifically, it found that complaints from customers with numerous social media followers were more likely to elicit responses from airlines than complaints from those who had few followers. Notably, the study also found that customers who mentioned other accounts (Twitter accounts) in their complaints were less likely to elicit responses - a manifestation of the bystander effect, where the complaining tweet is not just directed at the airline alone, but also directed at other twitter users. [12].

Social media use does not stop at the customer service level. Companies have implemented social media technologies internally to socialize employees and motivate newly hired members of younger generations. How companies that have implemented internal social media have gained value by encouraging employees to share their knowledge with other employees has been investigated [24]. This study showed that the use of social media technologies within organizations helped employees acquire accurate information from the correct people, enabling them to "know who knows what and who knows whom in the work place" [24]. In addition, it found that social media helped make the most knowledgeable employees more visible to their colleagues.

IS research papers have also investigated the phenomenon of enterprise-hosted online communities. For example, the impact of user- and marketergenerated content on consumer purchasing behavior has been examined [11]. This study analyzed the company Facebook Fan Subscriptions (FFS's) where its representatives interact with customers and customers can interact with each other. It found that high levels of engagement and interaction with the company via the fan page caused customers to increase their purchasing behavior. The study also discovered that user-generated content was more powerful than marketer-generated content when it came to influencing future customers [11]. This helps explains why word of mouth (WOM) has a strong effect on others.

The effects of customer activities and participation on firm-hosted fan pages on social media platforms such as Facebook have also been examined [37]. This study concluded that as customers' levels of engagement on firm social media pages increased, their profitability to the firms increased as well. This finding highlights the importance of firms engaging with customers on social media.

IS scholars have investigated many areas that fall under the category of "Enterprise-Controlled Social Media in Business Context". Their work helps understand how enterprises use social media to provide customer service and how those enterprises interact with consumers. IS research papers in this category 
also examined how enterprises use social media to manage communications between employees which helped increase the overall knowledge of employees.

\section{Enterprise-Controlled Social Media in Non-Business Contexts}

Papers in this category investigated how companies and organizations have implemented social media technologies to enhance activities that are not related directly to their core business aims. In other words, these papers considered enterprise-controlled social media in non-business contexts. For example, how organizations use enterprise-controlled social media to socialize employees has been investigated [23]. This study adopted the technology affordance theory to identify the affordances of enterprise-controlled social media and the actualized features actors are able to utilize. Its analysis showed that enterprise-controlled social media have both positive and negative affordances. These findings could help managers understand both the positive and negative impacts of enterprise-controlled social media on employees.

The impact of social media on internal organizational communication is an important phenomenon. Previous research has investigated how the adoption of social media enables organizations to manage internal communication. by applying the ambidexterity theory, the study examined how social media features allow enterprises to see employees' different perspectives through visible communications [16]. Additionally, the impact of social media on knowledge sharing has been examined through the lens of the communication visibility theory [24]. This study found that when invisible communication between two parties within an organization became visible to third parties, the third parties increased their knowledge of the subject matter. In addition, its analysis showed that the owners of the communicated messages became more visible to others. This study also revealed that the visibility of social media communication reduced redundant work because users were able to track the work others had completed [24].

Lastly, IS studies have examined social media and information technology (IT) culture conflicts within organizations. Organizations that implement social media technologies in the workplace face potential culture conflicts between employees and organizational IT policies. How organizations can address social media-related IT culture conflicts has been investigated [20]. Organizations need to acknowledge potential conflicts between employees' perceptions of social media values and organizations' IT values and seek, to the extent possible, to bring these different perceptions into alignment, thereby helping employees recognize the value of organizations' IT [20]. Additionally, the importance of reminding employees about organizations' IT policies, while pointing out that organizations must embrace social media as part of their employees' daily activities is highlighted.

To summarize, IS research papers in this category examined the phenomena of using social media within the enterprise and how it affect employees when it comes to compliance with existing IT culture and policies. Also, those papers examine how enterprises balance between applying IT polices and attracting new young employees to the work environment.

\section{Individual-Controlled Social Media in Non-Business Contexts}

There is a total of 27 articles covering various domains in which social media control belongs to individuals in non-business contexts. This area is the most researched category in this literature review. For example, how people organize themselves during crises has been a frequently investigated theme [33] [34] [45][49] [25] [44] [43]. Studies have examined how people have begun leveraging IT and social media applications to manage and survive during various types of crises - from natural disasters to terrorist attacks; these studies have considered how social media applications have facilitated survival-related activities by enabling people to communicate instantly, flexibly, and without relying on traditional centralized crises management organizations. Research has shown that social media applications not only help people organize during crises, but their features also allow people to express their emotions following crises and tragic events [41] [39]. Social media platforms offered people a space where they can fulfil their psychological needs when it comes to emotion expression.

Social media applications enable people to generate, share, and spread news and information across the globe faster than the mainstream media, which has limited access. They have essentially made individuals into independent news agencies who no longer depend on the mainstream media to get or disseminate their news [38]. In fact, the mainstream media often turns to social media to get news and information from individuals at the heart of a story. Individuals can now report instantly from wherever news is happening even before the mainstream media can arrive; the speed and flexibility of social media thus enables individuals to outperform the mainstream media. These features of social media were instrumental in the Arab Spring 2011, which was 
largely organized and managed using different social media applications. Indeed, these applications facilitated the activities of protestors-from spreading news regarding what was happening in different locations to relocating crowds and protesters [35].

Many of the studies in this category also investigated the issue of individual privacy on social media. As awareness of privacy issues on social media has increased, understanding the value people place on their social media privacy-and what they are willing to exchange for their privacy-has become increasingly important. Studies of privacy and information discloser on social media have found that as long as the perceived benefits outweigh the costs, people will continue to reveal personal information on social media [22] [14].

Studies in this category have also considered the differences between men and women in social media use [47] [21]. They report that men and women have different perspectives regarding what they can get from using social media. They have shown that when it comes to social media applications men are goaloriented-using the applications they believe will help them achieve their goals-whereas women are more emotion- and relationship-orientated-using social media applications to reinforce existing relationships and express their emotions.

The last theme considered by studies in this category is online communities. IS studies have examined online communities to understand what motivates members to participate and continue returning [2] [46]. These studies have shown that although online communities "such as travel focused forum" and "career focused forum" existed long before social media platforms became popular. As social media platforms grew on the Internet, they have started to absorb these other online communities.

To recap, numerous IS studies have investigated the social media phenomena and how it has changed people's behavior. Many IS papers examined how people use social media to organize themselves during crises to reduces the risk of crises outcomes due to chaos. With the use of social media during crises and other events, individuals are becoming independent sources of valuable news and information that could make huge difference during those events. Next section will show how social media enabled individuals to have some control over social media when debating business related issues.

\section{Individual-Controlled Social Media in Business Contexts}

Studies in this category investigated the use of individually-controlled social media in business contexts. Such individuals use social media to discuss business matters such as product reviews or issues related to specific products, or to engage in general discussions about their loved product or brand. As a form of social media, expert blogs and product communities are valuable to organizations, since the content generated by them can provide helpful feedback and overall user experiences. Indeed, research has shown that the content of product reviews can affect the value of companies by changing consumers' perceptions of the companies and the reviewed products [27] [5]. What occurs on social media can also impact firms' market values; thus, companies can no longer ignore the power of social media. Research has shown that companies must invest in social media to help protect their images among consumers [28]. This study also found that companies who manage their images on social media will enjoy greater brand awareness, which will be reflected in their bottom line [29].

As mentioned earlier, WOM is an interesting social media-based phenomenon that impacts consumers' decisions in terms of service and product recommendations. IS studies investigating how consumers react to online recommendations in the prepurchase phase have shown that interactions between consumers could help predict the future of products and serve as early indicators of how specific products will perform in terms of sales [31] [29]. WOM on social media, however, is not always associated with positive impacts. For example, research has shown that social media buzz regarding albums has a negative impact on music sales and, overall, traditional media (radio play) has a stronger positive affect on music sales than social media [7].

In summary, IS scholars studied how companied interacted with individual's vis social media regarding a product review, product suggestions and product promotions. Those activities occurred on social media platform where individuals controlled the conversation.

\section{Neutral Control and Context in Social Media Research}

For Studies in this category investigated phenomena related to both individual- and organization-controlled social media in general contexts. For example, how engagement affects online communities has been examined [36] [9]. In the context of online community engagement, one study examined the association between enjoyment and 
social media, finding both positive and negative associations, and highlighting the importance of examining how social media users perceive enjoyment [42].

Predicting user behavior on social media is important for understanding interactions between people and social media. One study found that affective commitment and continuous commitment are good indicators of social media users' behavior [6] Commitment itself can indicate the quality of a social media platform, demonstrating that platforms that lose the commitment of their users will fail and exit the market.

The final study in this category justified examining social media platforms irrespective of control and context [19]. It investigated the psychological needs that motivate the use of social media platforms, applying psychological theories to identify the needs that use of social media platforms satisfy.

To recap this category, IS scholars looked at how people use social media on their daily life and study how users of social media perceived enjoyment over social media. Predicting social media users behavior based on their activities on social media is another area that have been examined by IS scholars.

\section{Potential Gap}

Using the criteria of social media control and context, this literature review divided IS studies of social media into five categories. Each category encompassed different themes and domains. This categorical framework helps future social media researchers by identifying how current research fits into the social media research and where research gaps that may require further investigation exist.

For example, a potential gap exists in current research regarding individual-controlled social media in business contexts. IS studies have yet to fully consider the current phenomenon whereby consumers organize themselves using social media to impact companies' decisions regarding specific products or brands. Leveraging the power and flexibility that social media afford, consumers can now boldly voice their opinions to companies at little or no cost to themselves. Indeed, companies have found themselves in situations where they lose power over their consumers and have no choice but to listen to and negotiate with their customers. Thus, social media platforms have given consumers power that companies cannot ignore.

The phenomena where consumers and customers organize themselves using social media platforms to impact companies' decisions yet to be examined. This area has a potential for future IS research to examine the power consumers gained using social media.
Consumers are leveraging social media platforms to gain as much power as they could against enterprises and companies. These phenomena is worth to be investigated which could yield an interesting finds.

\section{Limitation:}

Though an acceptable practice, this research focused on papers published in top IS (the basket of eight) journals. Additional research may expand the literature review to include articles in a broader swath of journals outside of the basket. Even given this deeper review, however, we would expect to find similar results with articles varying on level of control and content.

\section{References}

[1] Ahmed, Ashir, Scheepers, Helana, and Stockdale, Rosemary. "Social Media Research: A Review of Academic Research and Future Research Directions (Book Review)." Pacific Asia Journal of the Association for Information Systems (2014): 21-37.

[2] Bateman, Patrick J., Peter H. Gray, and Brian S. Butler. "Research note - the impact of community commitment on participation in online communities." Information Systems Research 22.4 (2011): 841-854.

[3] Cao, J., Basoglu, K., Sheng, H., \& Lowry, P. B. “A systematic review of social networking research in information systems". Communications of the Association for Information (2015), Systems, 36(1).

[4] Chen, Wenshin, and Hirschheim, Rudy. "A Paradigmatic and Methodological Examination of Information Systems Research from 1991 to 2001." Information Systems Journal 14.3 (2004): 197-235. Web.

[5] Chau, M. C. L., and Jennifer Xu. "Business intelligence in blogs: Understanding consumer interactions and communities." MIS quarterly (2012).

[6] Chen, A., Lu, Y., Chau, P. Y., \& Gupta, S. (2014). Classifying, measuring, and predicting users' overall active behavior on social networking sites. Journal of Management Information Systems, 31(3), 213-253.

[7] Dean DL, Lowry PB, Humphreys S. Profiling the research productivity of tenured information systems faculty at US institutions. MIS Quarterly. 2010 Mar 1;35(1):1-5.

[8] Dewan, Sanjeev, and Jui Ramaprasad. "Social media, traditional media, and music sales." Mis Quarterly 38.1 (2014). 
[9] Dong, John Qi, and Wu, Weifang. "Business Value of Social Media Technologies: Evidence from Online User Innovation Communities." Journal of Strategic Information Systems 24.2 (2015): 113-127. Web.

[10] Germonprez, Matt, and Dirk S. Hovorka. "Member engagement within digitally enabled social network communities: new methodological considerations." Information systems journal 23.6 (2013): 525-549.

[11] Ghonim, W. (2012). Revolution 2.0: The power of the people is greater than the people in power: A memoir. houghton Mifflin harcourt.

[12] Goh, Khim-Yong, Cheng-Suang Heng, and Zhijie Lin. "Social media brand community and consumer behavior: Quantifying the relative impact of user-and marketergenerated content." Information Systems Research 24.1 (2013): 88-107.

[13] Gunarathne, Priyanga, Rui, Huaxia, and Seidmann, Abraham. "When Social Media Delivers Customer Service: Differential Customer Treatment in the Airline Industry." MIS Quarterly 42.2 (2018): 489-520. Web.

[14] Gunarathne, Priyanga, Rui, Huaxia, and Seidmann, Abraham. "Whose and What Social Media Complaints Have Happier Resolutions? Evidence from Twitter." Journal of Management Information Systems 34.2 (2017): 314-340. Web.

[15] Hey Tow, William Newk-Fon, Peter Dell, and John Venable. "Understanding information disclosure behaviour in Australian Facebook users." Journal of Information Technology 25.2 (2010): 126-136.

[16] Hootsuite.com

[17] Huang, Jimmy, João Baptista, and Sue Newell. "Communicational ambidexterity as a new capability to manage social media communication within organizations." The Journal of Strategic Information Systems 24.2 (2015): 49-64.

[18] Hu, Tao, William J. Kettinger, and Robin S. Poston. "The effect of online social value on satisfaction and continued use of social media." European Journal of Information Systems 24.4 (2015): 391-410.

[19] Kaplan, Andreas M., and Michael Haenlein. "Users of the world, unite! The challenges and opportunities of Social Media." Business horizons 53.1 (2010): 59-68.

[20] Karahanna, E., Xu, S. X., Xu, Y., \& Zhang, N. A. (2018). The Needs-Affordances-Features Perspective for the Use of Social Media. Management Review, 26(2), 298-310.

[21] Koch, Hope, Dorothy E. Leidner, and Ester S. Gonzalez. "Digitally enabling social networks: resolving IT-culture conflict." Information Systems Journal 23.6 (2013): 501-523.
[22] Krasnova, H., Veltri, N. F., Eling, N., \& Buxmann, P. (2017). Why men and women continue to use social networking sites: The role of gender differences. The Journal of Strategic Information Systems, 26(4), 261-284

[23] Krasnova, H., Spiekermann, S., Koroleva, K., \& Hildebrand, T. (2010). Online social networks: Why we disclose. Journal of information technology, 25(2), 109-125.

[24] Leidner, Dorothy E., Ester Gonzalez, and Hope Koch. "An affordance perspective of enterprise social media and organizational socialization." The Journal of Strategic Information Systems 27.2 (2018): 117-138.

[25] Leonardi, Paul. "Ambient Awareness and Knowledge Acquisition: Using Social Media to Learn 'Who Knows What' and 'Who Knows Whom.'” MIS Quarterly 39.4 (2015): 747-762. Web.

[26] Leong, Carmen, Pan, Shan, Ractham, Peter, and Kaewkitipong, Laddawan. "ICT-Enabled Community Empowerment in Crisis Response: Social Media in Thailand Flooding 2011." Journal of the Association for Information Systems 16, no. 3 (March 1, 2015): 174-212.

[27] Lowry PB, Gaskin J, Humpherys SL, Moody GD, Galletta DF, Barlow JB, Wilson DW. Evaluating journal quality and the association for information systems senior scholars' journal basket via bibliometric measures: Do expert journal assessments add value?. MIS quarterly. 2013 Dec 1:993-1012.

[28] Liu, Franklin, and Michael D. Myers. "An analysis of the AIS basket of top journals." Journal of Systems and Information Technology 13.1 (2011): 5-24.

[29] Luo, X., Zhang, J. J., Gu, B., \& Phang, C. (2013). Expert blogs and consumer perceptions of competing brands. MIS Quarterly, 41(2), 371-395.

[30] Luo, Xueming, Jie Zhang, and Wenjing Duan. "Social media and firm equity value." Information Systems Research 24.1 (2013): 146-163.

[31] Luo, Xueming, and Jie Zhang. "How do consumer buzz and traffic in social media marketing predict the value of the firm?." Journal of Management Information Systems 30.2 (2013): 213-238.

[32] Levy, Yair, and Timothy J. Ellis. "A systems approach to conduct an effective literature review in support of information systems research." Informing Science 9 (2006).

[33] Matook, Sabine, Susan A. Brown, and Johanna Rolf. "Forming an intention to act on recommendations given via online social networks." European Journal of Information Systems 24.1 (2015): 76-92. 
[34] Mingers, John. "The Paucity of Multimethod Research: a Review of the Information Systems Literature." Information Systems Journal 13.3 (2003): 233-249. Web.

[35] Ning Nan, and Yong Lu. "Harnessing the Power of SelfOrganization in an Online Community During Organizational crisis.(Report).” MIS Quarterly 38, no. 4 (December 1, 2014): 1135-1157.

[36] Oh, Onook, Agrawal, Manish, and Rao, H. Raghav. "Community Intelligence and Social Media Services: a Rumor Theoretic Analysis of Tweets During Social crises.(Report).” MIS Quarterly 37, no. 2 (June 1, 2013): 407-426.

[37] Oh, Onook, Chanyoung Eom, and H. Raghav Rao. "Research note-Role of social media in social change: An analysis of collective sense making during the 2011 Egypt revolution." Information Systems Research 26.1 (2015): 210223

[38] Ray, Soumya, Sung S. Kim, and James G. Morris. "The central role of engagement in online communities." Information Systems Research 25.3 (2014): 528-546.

[39] Rishika, R., Kumar, A., Janakiraman, R., \& Bezawada, R. (2013). The effect of customers' social media participation on customer visit frequency and profitability: an empirical investigation. Information systems research, 24(1), 108-127.

[40] Shi, Zhan, Huaxia Rui, and Andrew B. Whinston. "Content sharing in a social broadcasting environment: evidence from twitter." Available at SSRN 2341243 (2013).

[41] Stieglitz, Stefan, and Linh Dang-Xuan. "Emotions and information diffusion in social media - sentiment of microblogs and sharing behavior." Journal of management information systems 29.4 (2013): 217-248.

[42] socialbakers.com

[43] Senior Scholars Journal Review Quality Survey Association for Information Systems (AIS), https://aisnet.org/page/SeniorScholarSurvey/Senior-ScholarsJournal-Review-Quality-Survey.htm.

[44] Tim, Y., Pan, S. L., Ractham, P., \& Kaewkitipong, L. (2017). Digitally enabled disaster response: the emergence of social media as boundary objects in a flooding disaster. Information Systems Journal, 27(2), 197-232.

[45] Turel, Ofir, and Alexander Serenko. "The benefits and dangers of enjoyment with social networking websites." European Journal of Information Systems 21.5 (2012): 512-528.

[46] Tim, Y., Pan, S., Bahri, S., \& Fauzi, A. (2018). Digitally enabled affordances for community-driven environmental movement in rural Malaysia. Information Systems Journal, 28(1), 48-75.

[47] Vaast, E., Safadi, H., Lapointe, L., \& Negoita, B. (2017). Social Media Affordances or Connective Action: An Examination of Microblogging Use During the Gulf of Mexico Oil Spill. MIS Quarterly, 41(4), 1179-1206

[48] Wakefield, Robin, and Wakefield, Kirk. "Social Media Network Behavior: A Study of User Passion and Affect." Journal of Strategic Information Systems 25, no. 2 (July 2016): 140-156.

[49] Watts, Stephanie A., and Wei Zhang. "Capitalizing on content: Information adoption in two online communities." Journal of the Association for Information Systems 9.2 (2008): 3.

[50] Xl Shen, A., Lee, M. K., Cheung, C. M., \& Chen, H. (2010). Gender differences in intentional social action: weintention to engage in social network-facilitated team collaboration. Journal of Information Technology, 25(2), 152-169.

[51] Zheng, Yingqin, and Yu, Ai. “Affordances of Social Media in Collective Action: The Case of Free Lunch for Children in China." Information Systems Journal 26.3 (2016): 289-313. Web.

[52] Zuhair Khan, and Sirkka L Jarvenpaa. "Exploring Temporal Coordination of Events with Facebook.com." Journal of Information Technology 25, no. 2 (June 18, 2010): 137-151. 